\title{
Multicolour single molecule emission and excitation spectroscopy reveals extensive spectral shifts
}

\author{
Lukasz Piatkowski, $\uparrow^{a}$ Esther Gellings $\dagger^{a}$ and Niek F. van Hulst ${ }^{\star a b}$
}

Received 10th June 2015, Accepted 16th July 2015

DOI: $10.1039 / c 5 f d 00107 b$

\begin{abstract}
We explore the distribution and shape of single molecule spectra at room temperature, when embedded in a polymer host. Multicolour excitation and emission spectroscopy is implemented to capture the full inhomogeneous distribution. We observe dramatic spectral changes in a distribution of single quaterrylene diimide (QDI) molecules isolated in a PMMA matrix. The molecules are strongly blue shifted with respect to the ensemble absorption maximum and spread over a staggering $200 \mathrm{~nm}$ range. Despite these strong shifts, the shape of the emission spectra does not differ much between individual molecules. We demonstrate that a considerable number of molecules may be invisible in single molecule experiments, as they typically rely on only a single excitation wavelength, which predetermines which subensemble is probed in the experiment. Lastly, we make a first step towards single molecule excitation spectroscopy under ambient conditions, which allows us to determine the spectral range at which individual molecules absorb light most efficiently. We show how single molecule emission and excitation spectroscopies can complement each other and a combination of both techniques can help in understanding the origin of underlaying spectral properties of individual molecules.
\end{abstract}

\section{Introduction}

Single molecule (SM) experiments allow us to study a system of interest addressing one molecule at a time. Molecules are not isolated in nature, but are constantly interacting with their surroundings. This, together with an intrinsic molecular diversity, leads to the observation of inhomogeneities and dynamical processes that are usually concealed in the ensemble average. ${ }^{1,2}$

Depending on the system and photophysics of interest, different detection schemes are feasible. With very few exceptions, all SM methods rely on the

${ }^{a}$ ICFO-Institut de Ciences Fotoniques, The Barcelona Institute of Science and Technology, 08860 Castelldefels, Barcelona, Spain.E-mail: Niek.vanHulst@ICFO.eu

${ }^{b}$ ICREA-Institució Catalana de Recerca i Estudis Avançats, 08010 Barcelona, Spain

$\dagger$ These authors contributed equally to this work. 
detection of spontaneous emission. In cryogenic studies, the limited interactions between molecules and their local environment are exploited by measuring the typically lifetime-limited zero-phonon absorption lines. Under ambient conditions, the increased interactions with the environment lead to significantly broadened absorption lines as well as a drastic reduction in the photostability and number of photons emitted before photobleaching. As a consequence, at cryogenic temperatures SMs are commonly studied through both their fluorescence excitation and emission spectra, ${ }^{3-5}$ while at room temperature SMs are only routinely investigated through their fluorescence emission spectra. ${ }^{6,7}$

Spectral and dynamical variability including spectral shifts and blinking are observed irrespective of the chosen experimental parameters. ${ }^{8-12}$ They can be linked to changes in the chromophore itself (intrinsic) or the chromophores' local environment (extrinsic) that are mirrored in changes in the fluorescence emission. Intrinsic variability can for example be induced by intersystem crossing or conformational changes, whereas reasons for extrinsic changes include external fields and changes in the molecules' local environment. Especially in heterogeneous environments like polymers, individual molecules can interact with a manifold of chemically or physically distinct surroundings. Structural differences like the size, shape and the local surface composition of the polymer affect among other things the polarity, acidity and rigidity of the nanoenvironment. Depending on the degree and nature of the interaction, this may be reflected in more or less pronounced deviations from the molecule ensemble average spectral position and shape, quantum yield or lifetime. ${ }^{13-18}$ Dynamical changes in the behaviour of the molecules can be photoinduced, for example by a rearrangement of the host matrix around the molecule due to local heating ${ }^{14}$ or cis/trans conformational changes. ${ }^{19,20}$ Still, the trapping of molecules in a crystal matrix or a polymer film is generally beneficial. It increases the spectral stability, because it forces the molecules into a certain conformation and hinders the diffusion of oxygen to the molecule, resulting in a lower photobleaching rate. ${ }^{21-23}$

Spectral variability of SMs has been experimentally observed in a number of systems. A spectral distribution of $40 \mathrm{~nm}$ has been reported for fluorescent dyes based on the perylene diimide chromophore (structurally similar to QDI). ${ }^{\mathbf{1 4}, 15}$ In these cases, large spectral shifts have been explained in terms of a twisting of the chromophore core (intrinsic) as well as a varying local polymer environment (extrinsic). Spectral variations of nearly $100 \mathrm{~nm}$ have been reported for other systems such as green fluorescent proteins in $\mathrm{PVA}^{\mathbf{1 9}}$ and carbocyanine dye molecules adsorbed on bare glass. ${ }^{20}$

In this work, we investigated the spectral distribution of individual QDI (quaterrylene diimide) ${ }^{24}$ molecules embedded in a polymer host (PMMA) under ambient conditions. QDI molecules belong to the rylene dye family that has been extensively used in SM research over the past decade due to its high quantum efficiency and photostability. ${ }^{3,25-28}$ The absorption and emission wavelengths of rylene dyes can be tuned throughout the visible spectrum by adjusting the length of the perylene backbone. ${ }^{29}$ This determines the degree of $\pi$-electron delocalization and thus the electronic transition strength and energy. ${ }^{30}$

Here, we demonstrate the importance of utilizing a range of excitation wavelengths and detection bandwidths to access and probe the full spectral range of SMs. We show how the choice of excitation and detection wavelengths can put a 
bias on the measured spectral distribution for both SM emission and excitation spectroscopy.

\section{Results}

Environmental effects on the spectral properties of molecules can already be visible in ensemble experiments. That is why it is informative to first compare the ensemble absorption and emission spectra in solution to those in a polymer matrix, and get an indication of the spectral changes we might expect from SM experiments. We dropcast QDI/PMMA/toluene solution with a QDI concentration of $\sim 10 \mu \mathrm{M}$ onto a cover slip, let the solvent evaporate and subsequently measured the ensemble absorption and emission spectra. We compared the results to the QDI solution spectra measured with the same concentration.

In Fig. 1 we show the absorption and emission spectra of QDI in solution (shaded grey and red, respectively) and in a solidified PMMA film (solid blue and red lines). In both cases the emission spectra were measured using $633 \mathrm{~nm}$ excitation light derived from a HeNe laser. The inset shows the chemical structure of QDI.

In the solution absorption spectrum, we observed three bands corresponding to the electronic transition at $750 \mathrm{~nm}$ and two vibronic sidebands at $680 \mathrm{~nm}$ and $630 \mathrm{~nm}$. The solution emission spectrum is not the exact mirror image of the absorption spectrum as would be expected for chromophores that strictly follow Kasha's rule. The emission spectrum has only two bands at $785 \mathrm{~nm}$ and $860 \mathrm{~nm}$ and its interband intensity ratio differs from the corresponding interband ratio in the absorption spectrum.

The molecular ratio between the polymer and QDI is roughly 10/1. Given that the polymer molecules are approximately 100 times larger than QDI we could safely assume that at this concentration the QDI molecules mainly interact with the polymer and not with each other. The measured spectra show that the absorption of QDI in a PMMA film significantly differs in shape from the absorption in solution (see Fig. 1). The maximum intensity of the spectrum is shifted towards lower wavelengths by approximately $50 \mathrm{~nm}$ and lacks the spectral features of the vibronic progression. Correspondingly, we found that the emission spectrum of the dry film QDI was blue shifted by $\sim 20 \mathrm{~nm}$ with respect to the solution emission spectrum. However, its shape was largely unaltered.

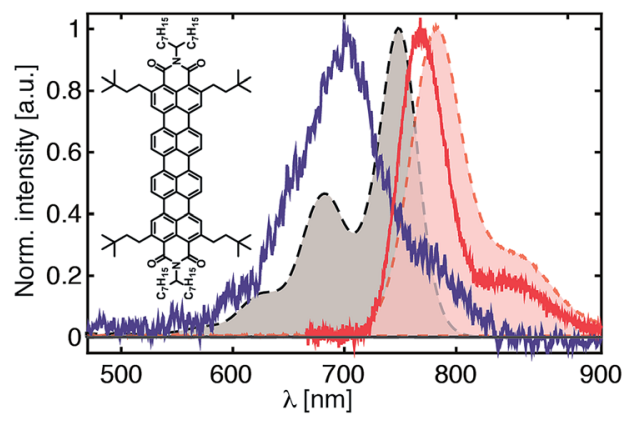

Fig. 1 Absorption and emission spectra of QDI in PMMA/toluene solution (shaded grey and red, respectively). Dry QDI/PMMA film absorption and emission spectra (solid blue and red lines). The chemical structure of QDI is shown alongside the spectra. 
It is important to note that the observed changes in the absorption spectra of the QDI film are reversible - when the dry film was dissolved in toluene again, the QDI solution spectrum was recovered.

It is clear that the local nano-environment induced by the proximity of the glass and/or polymer does alter the absorption and emission spectra of the QDI ensemble. The question that arises naturally from these observations is whether comparable changes in spectral shape and position can be found at the SM level. Do individual molecules embedded in PMMA mirror the shape and position of the ensemble dry film absorption and emission spectra, or does an induced spread of molecules with different electronic transition energies and different spectral shapes lead to the observed changes? With these questions in mind we now turn our attention to multicolour SM experiments.

\section{Multicolour excitation confocal spectroscopy}

For the SM experiments, samples with a density of about 1 QDI molecule per 2 $\mu \mathrm{m}^{2}$ were used to ensure that the molecules are well separated for the confocal detection. As the first step, a confocal image was recorded to locate molecules on the sample. Then the focal position was optimized based on the intensity of the fluorescence signal detected with an APD. We then acquired a series of five confocal images of the same sample area at excitation wavelengths ranging from
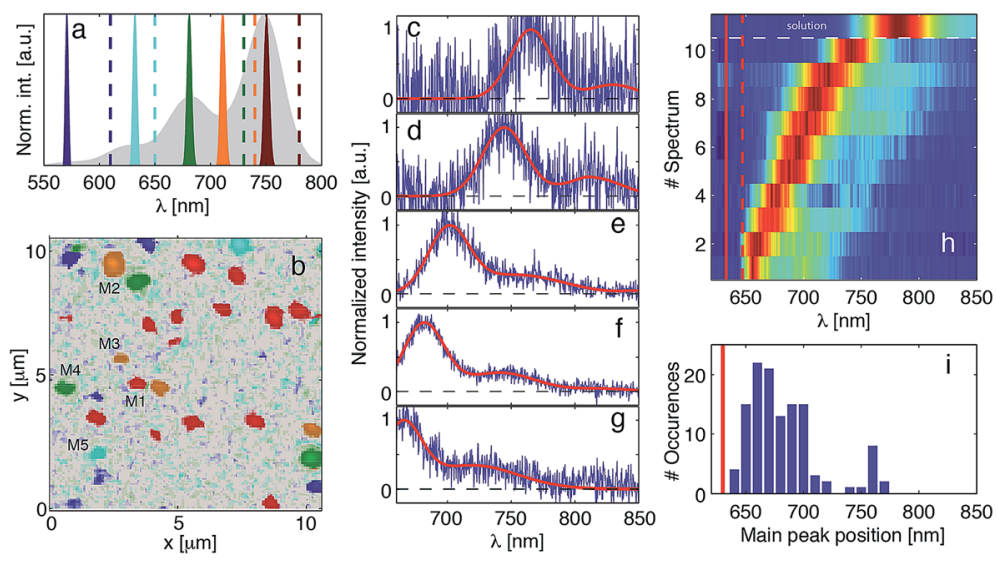

Fig. 2 (a) Concept schematic of the experiment. Coloured profiles and dashed lines represent the excitation wavelengths and the cut-off wavelengths of the corresponding long-pass filters, respectively. The grey shaded area represents the ensemble absorption spectrum of QDI in solution. (b) Zoom in on multicolour excitation confocal image composed of five individual confocal images obtained at the excitation wavelengths indicated in panel a. (c-g) SM emission spectra measured on five distinct molecules (M1 to M5 from top to bottom) numbered on the confocal image in panel $b$. The solid red lines represent the Gaussian fit to the measured emission spectra. (h) The spread of the emission spectra of individual molecules shown in the form of a 2D map. The top slice shows the emission spectrum of QDI in solution. Solid and dashed red lines indicate excitation $(633 \mathrm{~nm})$ and long-pass filter cut-off wavelengths $(650 \mathrm{~nm})$, respectively. (i) Histogram of the spectral positions for all (122) measured SM emission spectra. The spectral positions of individual molecules correspond to the position of the maximum intensity in their emission spectrum (maximum of the electronic transition). 
$750 \mathrm{~nm}$ to $570 \mathrm{~nm}$ (coloured spectra in Fig. 2a). For each excitation wavelength we used an appropriate long-pass filter with a cut-off wavelength spectrally as close as possible to the excitation wavelength, to maximize the fluorescence detection efficiency. If the molecules have a spread in absorption spectra, a different subset of molecules will get excited at each excitation wavelength so that information on the amount of excitable molecules at each wavelength can be extracted. We started on the red side with a confocal image using $750 \mathrm{~nm}$ wavelength excitation light. All molecules whose absorption spectrum overlapped with that wavelength appeared in this image. Subsequently we took a confocal image at $710 \mathrm{~nm}$ excitation. As the absorption spectrum of QDI is nearly $200 \mathrm{~nm}$ wide, the molecules that were detected at $750 \mathrm{~nm}$ excitation will also appear on this image. Additionally, however, we found extra molecules whose absorption spectrum was blue shifted so much that they could be excited at $710 \mathrm{~nm}$, but not yet at $750 \mathrm{~nm}$. We repeated this for subsequent excitation wavelengths towards the blue, to find all the subsets of molecules that start absorbing at each of the excitation wavelengths. We assigned a colour to these sub-ensembles, according to the colour of the excitation wavelength at which they first appear in a confocal image (not really corresponding to the VIS range). The molecules that were visible on a particular image were manually removed from all images acquired at shorter excitation wavelengths. Then, the average background was removed and the data smoothed with a Gaussian filter. Finally, the images were normalized to each other and overlaid. For better visibility of the molecules, the bottom $5 \%$ intensity on the images (residual background) has been coloured grey. The resulting multicolour excitation confocal image is shown in Fig. $2 \mathrm{~b}$. The variation in size between molecules simply results from the diversity in emission intensity.

We have experimentally checked that there were no molecules significantly redshifted with respect to the ensemble spectrum by measuring a confocal image at $800 \mathrm{~nm}$ excitation. Assuming that molecules can be detected when excited at the level of at least $10 \%$ of their absorption cross section, they would appear on a confocal image if their maximum absorption peak would reside at most $40 \mathrm{~nm}$ below the excitation wavelength (assuming that the spectral shape is largely unaltered). Thus we conclude that there were no molecules shifted by more than $10 \mathrm{~nm}$ towards the red.

The continuous appearance of molecules on the images, even as blue shifted as $570 \mathrm{~nm}$, indicates that the sample contains molecules with a large spread of electronic transition energies. To find out whether the observed spread is also associated with changes of the spectral shape and the vibrational band structure, we now turn to SM emission and excitation spectroscopy.

\section{Single molecule emission spectra}

We measured emission spectra of individual molecules on the same area of the sample upon excitation at $633 \mathrm{~nm}$. In panels c-g of Fig. 2 we show five exemplary emission spectra measured on the molecules that appeared at different excitation wavelengths and are numbered M1-M5 in Fig. 2b. We found that the emission spectra of molecules emitting on the red side near the QDI solution emission wavelength (Fig. 2c and d) were much weaker and much noisier. This was expected because at $633 \mathrm{~nm}$ excitation the absorption cross-section is very low for molecules with maximum absorption around $750 \mathrm{~nm}$ ( $15 \%$ of the maximum). 
Still, there was a continuous distribution of molecules absorbing at all intermediates, which became clear when we measured more emission spectra on different areas of the sample. We found that the position of the maxima of the emission spectra ranged from about $760 \mathrm{~nm}$ to the cut-off wavelength of our longpass filter at $647 \mathrm{~nm}$.

In panel h we show a series of $10 \mathrm{SM}$ emission spectra. In the top slice we show the emission spectrum of QDI in solution for comparison. A histogram showing the wavelength of the maximum emission for all measured molecules is shown in panel $i$. The positions of the maxima were determined using a Gaussian fit to the main peak of the emission spectrum. The distribution clearly peaks towards shorter wavelengths, close to where the excitation wavelength is. This is understandable, as the molecules that have their maximum absorption shifted towards the blue overlap with the excitation laser more strongly and thus appear brighter on the image. In the experiment we are naturally biased towards picking brighter molecules. This observation demonstrates that the choice of excitation and detection bandwidths determines which molecular sub-ensemble is probed most efficiently when the molecules show a large spectral distribution.

We found new molecules appearing when the excitation wavelength is moved towards the blue in the multicolour excitation confocal image (by nearly $200 \mathrm{~nm}$ ). We also found a distribution of emission spectra with nearly the same shape, while spreading over at least $100 \mathrm{~nm}$. These observations clearly indicate that the individual molecules keep their intrinsic spectral properties but experience an overall electronic shift.

\section{Towards excitation spectra of single molecules}

We have learned that the absorption spectrum of QDI molecules shifts together with the emission spectrum. Since the shape of the emission spectra did not appear to depend on the spectral position, we tentatively assumed that the shape of the absorption spectra would not vary significantly between individual molecules either. It is however desirable to find out whether the shape of the absorption spectra resembles that of QDI in solution or whether it is affected by the encapsulation in PMMA.

In the following step we therefore attempted to measure the excitation spectra of individual molecules. Fig. $3 \mathrm{f}$ shows the concept of this multicolour experiment. We recorded confocal images at different excitation wavelengths again but kept the long-pass filter in the detection path constant at $776 \mathrm{~nm}$ this time. This way the same portion of fluorescence emission was detected at each excitation wavelength. Therefore, for the same excitation power, the detected fluorescence intensity only depended on the molecule's absorption cross-section at the excitation wavelength. Variations in fluorescence intensity between different excitation wavelengths therefore directly yield the excitation spectrum of the molecule in this case. The measured confocal images are shown in Fig. 3a-e. The confocal images measured towards the blue excitation contain fewer and fewer molecules, which results from the fact that the red molecules have a very low absorption cross section at these wavelengths, whereas for the blue molecules the amount of detected fluorescence is very low. Examples of SM excitation spectra are shown in Fig. 3g-j (solid blue lines). The excitation spectrum shown in Fig. 3j corresponds to the molecule marked with a red circle in the confocal images. As a guide to the 

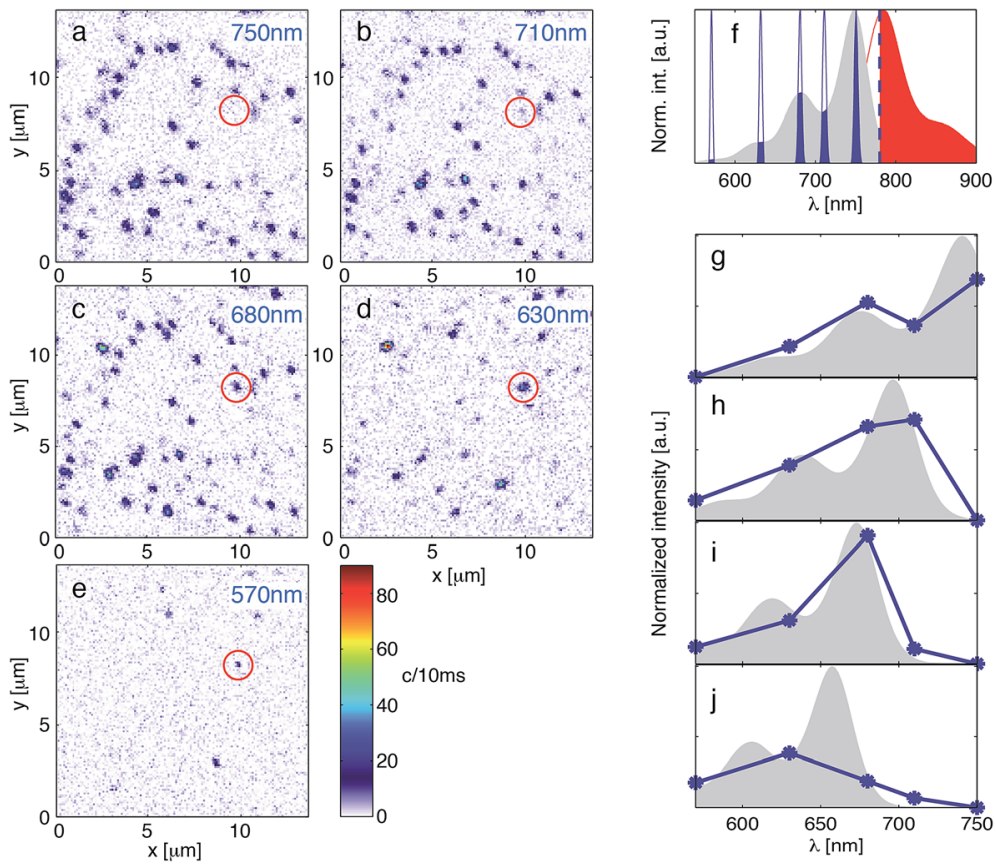

Fig. 3 (a-e) Confocal images measured at different excitation wavelengths, indicated in the right upper corner of each image, with the fluorescence signal detected with the same $776 \mathrm{~nm}$ long-pass filter. (f) Concept schematic of the experiment. The blue profiles represent the excitation wavelengths and the blue dotted line the cut-off wavelength of the long-pass filter. The red filled area indicates the detected part of the emission spectrum. $(g-j)$ The blue lines represent the SM excitation spectra reconstructed from the confocal images shown in panels a to e. For comparison we also show the absorption spectrum of QDI in solution (shaded grey) that has been spectrally blue shifted to match the measured SM excitation spectrum. The ensemble absorption spectra were shifted in the frequency domain and then translated back to the wavelength range to account for the difference in photon energy corresponding to different wavelengths.

eye, we added a QDI solution absorption spectrum, which has been spectrally shifted to match the measured excitation spectra (grey, shaded). The five measured experimental points were insufficient to precisely reproduce the spectral shape of the absorption spectrum. However, they were sufficient to determine the spectral position of the excitation spectra with about $20 \mathrm{~nm}$ precision and also to verify that the width of the excitation spectra matches that of the solution absorption spectrum. Looking at different molecules we found a large spread of spectral positions of about $100 \mathrm{~nm}$ for the excitation spectra.

\section{Quantitative analysis}

Upon careful inspection of the SM emission spectra we found that the separation between the electronic transition and the vibronic sideband, as well as their relative intensities, varied between individual molecules. In Fig. 4a we show the correlation between the spectral position of the main peak and the spectral separation between the electronic transition and vibronic sideband. We took into 

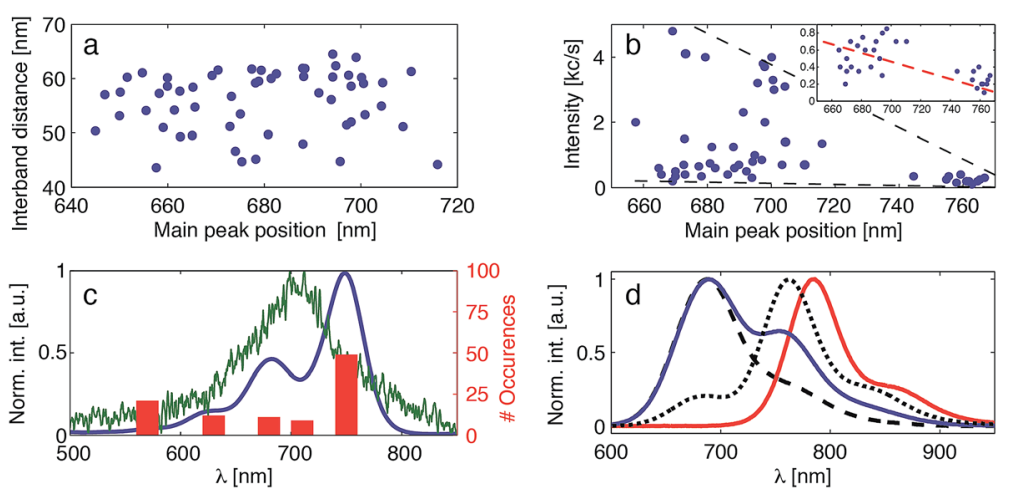

Fig. 4 (a) Correlation between the spectral position of the SM emission spectra and separation between the main electronic transition and the first vibronic band. (b) Correlation between the SM spectral position and intensity of the emission spectrum. The black dashed lines indicate the limits of the plotted distribution. The inset zooms into the low count range of the main panel with the red dashed line acting as guide to the eyes. (c) Comparison between the absorption spectrum of QDI film (green) and the distribution of the single QDI molecule spectral positions (red bars) based on the multicolour excitation confocal image shown in Fig. 2b. (d) Comparison between the QDI solution emission spectrum (red) and the reconstructed SM ensemble emission spectrum for two subpopulations of molecules: bright (dashed line) and dark (dotted line) ones (giving >300 and $<300$ counts per s, respectively, as shown in the inset of panel b). The solid, blue line represents the SM ensemble emission spectrum, taking into account all the molecules shown in panel b.

account all SM emission spectra for which we could clearly see the two bands and did not find any clear correlation of the interband separation and maximum wavelength at which the molecules emit. The majority of the molecules exhibited the same interband distance of about 50-60 nm even though they absorbed across a wavelength range of nearly $100 \mathrm{~nm}$.

From the analysis of the emission spectra of individual molecules taken from the multicolour excitation confocal image, we learned that picking up darker molecules yields emission spectra at the red side (towards $750 \mathrm{~nm}$ ), while brighter molecules emit towards the blue side. In Fig. $4 \mathrm{~b}$ we show the correlation between the maximum intensity of the emission spectrum and the spectral position of the main emission peak, when exciting at $633 \mathrm{~nm}$. Interestingly, the scatter plot has the shape of a wedge, as indicated with the thin, black dashed lines. The intensity spread for red molecules is much smaller than for blue molecules. This can be readily understood in terms of the transition dipole moment orientation and/or the internal quantum efficiency. When exciting at $633 \mathrm{~nm}$ we were more sensitive to blue molecules. Even not so efficient molecules and molecules whose transition dipole moment is not aligned well with the polarization of the excitation light would be visible on the image and bright enough to measure their emission spectrum. For the red molecules on the other hand, as they were excited less efficiently in our experiment, we were mainly sensitive to the brightest ones that had high quantum efficiency, and for which the transition dipole moment orientation matched the excitation light polarization, making the distribution much narrower. After looking closely at the low intensity sub-ensemble of molecules (see inset) we indeed found that the spread of intensities is similar for 
all molecules and we saw a clear linear dependence between the brightness and the spectral position. This dependence illustrates yet again that SM experiments are highly biased by the excitation wavelength, especially when only the bright sub-population is sampled.

Finally, from all our experiments on SMs we have found a large distribution of molecules absorbing and emitting at various wavelengths. We can thus reconstruct ensemble excitation and emission spectra from the SM results and tentatively compare them to the absorption and emission spectra measured on the QDI film. In Fig. 4c we show the absorption spectrum of the dry QDI film (green) together with a histogram (red), which shows occurrences of molecules starting to absorb at the five different excitation wavelengths. The histogram was constructed, taking into account molecules from the multicolour excitation confocal images (see Fig. 2b). The number of molecules that we have measured is insufficient to precisely rebuild the ensemble absorption spectrum of dry film. However, the extent of the distribution is well represented. For comparison, we also show the absorption spectrum of QDI in solution (blue). We note that multicolour excitation confocal microscopy with changing long-pass filters was the only unbiased experiment with respect to excitation/detection wavelength choice that we have performed.

In Fig. $4 \mathrm{~d}$ we show the reconstructed emission spectrum of the SM ensemble (blue) in comparison to the dry film emission spectrum (red). The SM ensemble spectrum was constructed by adding up normalized solution emission spectra that are shifted according to Fig. $4 \mathrm{~b}$. The SM ensemble emission spectrum is clearly blue shifted with respect to the dry film spectrum. This is in line with the fact that in the experiment we are biased towards brighter molecules $(633 \mathrm{~nm}$ excitation). If we only take into account bright molecules ( $>300$ counts per $s$ ), the reconstructed SM ensemble emission spectrum is strongly blue shifted (black dashed line), whereas if we take only the darker molecules ( $\leq 300$ counts per s) we find that the SM ensemble emission spectrum has nearly the shape of the QDI solution emission (black dotted line).

\section{Discussion}

From the multicolour excitation confocal images we have learned that QDI molecules embedded in a PMMA matrix can start absorbing anywhere between 570 and $750 \mathrm{~nm}$. The shifts in the absorption of up to $200 \mathrm{~nm}$ away from the solution absorption maximum $(750 \mathrm{~nm})$ are quite dramatic, especially when realising that they correspond to changes in the electronic transition energy of about 25\%.

Interestingly, we found that the shapes of the emission spectra of molecules absorbing at various wavelengths were nearly identical (Fig. 2c-g). The position of the first vibrational band with respect to the main electronic transition varied between 45 and $65 \mathrm{~nm}$, but there was no clear correlation between the inter-band distance and the wavelength at which the molecules emit. This indicates that the intramolecular coupling between vibrational modes and the electronic transition was largely unaltered. As the vibrational modes are quite sensitive to structural changes of the molecule, we concluded that all molecules must have a very similar conformation.

The spectral distribution in the measured SM excitation spectra shown in Fig. 3 is in full agreement with the spread observed in the measured emission 
spectra. The excitation spectra comprise only 5 points. While this was not sufficient to precisely determine the shape of the excitation spectra, the overall shape and spectral width still generally agree with the QDI solution absorption spectrum (shaded grey). It would evidently be highly beneficial to be able to measure excitation spectra of individual molecules fast and with high spectral resolution under ambient conditions. Only recently we have developed an experimental approach based on the broadband interferometric excitation that enables fast measurement of excitation spectra of single molecules at ambient conditions with high spectral resolution. ${ }^{31}$ Correlations between the excitation and emission spectra will undoubtedly shed new light on many fundamental intra- and intermolecular processes.

Based on our experiments the exact reason for the observed strong spectral variability among the individual molecules is hard to pinpoint. However, we know that the extent of $\pi$ electron delocalization in the rylene backbone determines the absorption/emission wavelengths and thus one possible explanation is that the conjugation in the backbone is truncated differently for individual molecules. In rylene dyes there is a $100 \mathrm{~nm}$ bathochromic shift for each attached naphthalene group. The shorter backbone homologues of QDI, like PDI and TDI, have their absorption maxima in solution at approximately 560 and $660 \mathrm{~nm}$, respectively. Importantly, they all have the same shape of the absorption spectrum. It thus seems plausible that either the change in the conformation and/or interactions with the polymer leads to a different degree of truncation of conjugation and to the observed distribution. It could either be the long hydrocarbon chains folding onto the backbone or the proximity of the polymer interfering with the conjugation. The unchanged emission and excitation spectra (the latter at least in terms of the width) would support this.

To check this hypothesis we repeated the experiments for QDI molecules spincoated on a glass coverslip without any polymer. Unfortunately, bleaching, strongly reduced observation time and much enhanced blinking dynamics did not allow us to draw any conclusions.

Hofkens et al. showed that substitutions in the bay region (middle region of the naphthalene backbone) of PDI based molecules may lead to large twists of the naphthalene backbone. However, twists reaching tens of degrees lead to spectral shifts of the emission spectra of only up to $40 \mathrm{~nm} .{ }^{14}$ On the one hand, in PDI (shorter homologue of QDI) without any substitutions in the bay area the flat conformation was calculated to be the most favourable one. On the other hand, the twist of the naphthalene backbone is associated with the loss of the fine structure in the vibrational progression. Even though QDI does not have any bay substitutions, we did observe a smoothing of the vibrational progression in the emission spectra of many measured molecules. Perhaps minor intrinsic or polymer induced twisting of the backbone also play a role in our studies.

Our results clearly demonstrate that SM experiments can be highly biased towards sub-ensembles in the case of a large spectral heterogeneity among the molecules. For QDI, the fact that the spread is nearly $200 \mathrm{~nm}$ broad combined with the choice of a particular wavelength (excitation or detection) might result in completely failing to capture part of the SM ensemble. It is of fundamental importance to keep this in mind for any SM experiment, because reliance on the ensemble solution spectrum for choosing appropriate excitation and detection wavelengths may lead to highly biased results. 
Both the multicolour excitation confocal images and the emission spectroscopy experiments provided information on the relative amount of molecules absorbing and emitting at specific wavelengths. We intuitively built up SM ensemble spectra and compared them to the absorption and emission spectra of QDI in solution, as shown in Fig. 4c and d. The spread of absorption wavelengths was well represented but we did find a discrepancy in the number of molecules absorbing at specific wavelengths. Our statistics were quite limited ( $\sim 100$ molecules) but it seems that there was about a 50/50 ratio of molecules absorbing around $750 \mathrm{~nm}$ to molecules absorbing at all other wavelengths, whereas the dry QDI film suggested that the distribution should peak around $700 \mathrm{~nm}$. The origin of this difference is unclear, but we cannot really rule out changes in the absorption cross section for spectrally distinct molecules.

The SM ensemble emission differed significantly from the dry QDI film emission spectrum. This difference is easy to understand because the experiment is highly biased towards molecules absorbing in the blue upon excitation at 633 $\mathrm{nm}$. In fact we could spectrally tune the SM ensemble emission spectrum by simply taking into account molecules of different brightness and consequently of different spectral position (see Fig. 4d). Furthermore, a reasonable number of the molecules whose fluorescence does not significantly stand out against the background might simply be ignored in the analysis. Lastly, changes in the quantum efficiency of the molecule with the spectral position cannot be completely excluded.

\section{Conclusions}

In this work we have addressed spectral changes in absorption and emission spectra of SMs embedded in a polymer matrix. We have found that QDI molecules exhibit a nearly $200 \mathrm{~nm}$ broad distribution of the position of the absorption and emission spectra, shifted towards lower wavelengths from the maximum absorption of QDI in solution. All molecules show a similar spectral shape but differ significantly in electronic transition energy.

We have demonstrated that any SM experiment may lead to biased results depending on the choice of excitation and detection wavelengths when the investigated molecules exhibit a large spectral variability. In extreme cases, part of the SM population may not be detected at all.

Due to an interplay between numerous intrinsic and extrinsic causes for spectral shifts, the spectral variability among SMs is hard to predict. Multiple wavelength excitation should therefore be utilized to find the full bandwidth of spectral positions of SMs on the sample.

Finally, being able to measure excitation spectra of individual molecules fast and with high spectral resolution would expand our knowledge of fundamental processes driving chemistry at the SM level. In particular, correlations between excitation spectra and other observables such as quantum efficiency, emission spectra, blinking dynamics and conformation might be extremely insightful. Moreover, knowledge of the position of the absorption and emission spectra is of fundamental importance for novel experimental approaches where for instance plasmonic enhancement is used on either absorption or emission of individual molecules. $^{32,33}$ 


\section{Experimental}

\section{Experimental setup}

A laser beam was derived from either a white light source (SuperK, NKT) or a HeNe laser. The laser beam was cleaned up using an appropriate band-pass filter and propagated colinearly into an inverted microscope (Observer D1, Zeiss). Inside the microscope, the laser light was reflected from a 50/50 beamsplitter and focused to a diffraction-limited spot onto the sample with a high numerical aperture oil-immersion objective (1.3 NA, 100×, Zeiss Fluar). The fluorescence from the sample was collected through the same objective and then either sent to a spectrometer equipped with an electron-multiplying charge-coupled device (EMCCD, Newton, Andor) camera or focused onto an avalanche photo-diode (APD, Perkin-Elmer) that allows confocal optical imaging of the sample. Longpass filters were placed in the detection path to block the reflected laser light. The sample was placed onto a piezo-controlled stage that enabled precise positioning and scanning. A shutter was positioned in front of the microscope to prevent sample illumination in between measurements.

The laser power was typically set in the range $1-4 \mu \mathrm{W}$ for a single beam at the sample position. For an average molecule 2000-3000 counts per s were detected, of which about $\sim 200$ counts per s was background count. Background count included the dark counts of the APD ( $\sim 60$ counts per $s)$ and residual stray light above the cut-off wavelength of the long-pass filter.

All the experiments were performed at least twice to ensure reproducibility.

\section{Sample preparation}

The experiments were performed on single quaterrylene diimide (QDI) molecules derived from the rylene dye family and obtained from the Müllen group (Max Planck Institute for Polymer Research, Mainz, Germany).

The samples were prepared by dissolving QDI molecules at a $\sim 0.1-1 \mathrm{nM}$ concentration in a poly(methyl methacrylate)-toluene solution (PMMA/toluene mixture $\sim 1 \% \mathrm{w} / \mathrm{v}$ ). Approximately $50 \mu \mathrm{L}$ of the solution was then spin-coated onto a $\mathrm{nr} 1 \mathrm{microscope}$ cover slip for $60 \mathrm{~s}$ at a spinning rate of $2000 \mathrm{rpm}$. Microscope cover slips were cleaned by immersing them in a piranha solution $(1: 2$ ratio hydrogen peroxide to sulfuric acid) for $\sim 30$ minutes, then rinsed with deionized water and blow-dried with $\mathrm{N}_{2}$. We found this procedure yielded no or very little contamination on the cover slips.

\section{Absorption and emission spectra of the QDI ensemble}

The absorption spectrum of QDI in solution was measured using a commercial spectrophotometer (NanoDrop 2000, Thermo Scientific). The absorption spectrum of the dry QDI film was measured using the experimental setup described above. A drop of QDI solution was placed onto a cover slip and allowed to dry. White light, derived from the built-in halogen lamp of the microscope, was transmitted through the sample and sent into the spectrometer. A reference white light spectrum was always measured alongside the dried QDI spot.

We used the same setup to measure the emission spectra of both dry QDI and QDI in solution. For the latter we placed a drop of the solution onto the cover slip 
and measured the emission spectrum directly afterwards. Both emission spectra were measured using $633 \mathrm{~nm}$ excitation light derived from a HeNe laser.

\section{SM emission spectra}

SM emission spectra were measured exciting at $\sim 633 \mathrm{~nm}$ with a HeNe laser. The laser light was cleaned up with a 632/11 band-pass filter and blocked with a 647 $\mathrm{nm}$ LP in front of the APD and spectrometer. The filters in front of the spectrometer were chosen as close to the excitation wavelength as possible in order to maximize the detectable spectral range.

After taking the confocal image, the background spectrum was taken with the EMCCD on the sample away from the molecules without changing the focus. Then, individual molecules were placed in the laser focus to record their emission spectra. Typically, 20 background-corrected emission spectra were recorded in a kinetic series with an integration time of $1 \mathrm{~s}$ per spectrum. The spectra before photobleaching or spectral jumping were averaged per molecule to improve the signal-to-noise ratio. The gain of the used EMCCD camera was set to 200. The quantum efficiency of our camera reaches its maximum around $700 \mathrm{~nm}$, and its variation in the spectral range of $600-800 \mathrm{~nm}$ is less than $8 \%$.

\section{Multicolour excitation confocal images}

Multicolour excitation confocal images were acquired in two different schemes. In the first approach the confocal images were measured for a specific set of excitation and detection long-pass filters. In the second approach the long-pass filter was fixed and only the excitation wavelength was tuned. In both schemes, the confocal fluorescence images were recorded with $5 \mathrm{~nm}$ broad excitation bands derived from an ultrabroadband laser (SuperK, NKT) equipped with an acoustooptic tunable filter. A narrowband band-pass filter matching the excitation wavelength was placed in the beam to ensure that no other wavelengths were reaching the sample.

In the first experiment, the excitation wavelengths were 570, 630, 680, 710 and $750 \mathrm{~nm}$ with respectively a $610 \mathrm{LP}, 650 \mathrm{LP}, 730 \mathrm{LP}, 740 \mathrm{LP}$ and $776 \mathrm{LP}$ filter (schematically indicated in Fig. 2a). In the second experiment we used the same excitation wavelengths while keeping the $776 \mathrm{~nm}$ long-pass filter for all acquired images (schematically shown in Fig. 3f). The images have a size of $20 \times 20 \mu \mathrm{m}$ with $200 \times 200$ pixel resolution and an acquisition time of $10 \mathrm{~ms}$ per pixel.

\section{Acknowledgements}

L. P. acknowledges financial support from the Marie-Curie International Fellowship COFUND and the ICFOnest program. E. G. acknowledges financial support from the Erasmus+ program. This research was funded by the European Commission (ERC Advanced Grant 247330-Nano Antennas), Fundació CELLEX (Barcelona) and Plan Nacional project FIS2012-35527.

\section{Notes and references}

1 W. E. Moerner, J. Phys. Chem. B, 2002, 106, 910-927.

2 B. Kozankiewicz and M. Orrit, Chem. Soc. Rev., 2014, 43, 1029-1043. 
3 R. Korlacki, M. Steiner, H. Qian, A. Hartschuh and A. J. Meixner, ChemPhysChem, 2007, 8, 1049-1055.

4 W. E. Moerner and L. Kador, Phys. Rev. Lett., 1989, 62, 2535-2538.

5 M. Orrit and J. Bernard, Phys. Rev. Lett., 1990, 65, 2716-2719.

6 P. Tchenio, A. B. Myers and W. E. Moerner, Chem. Phys. Lett., 1993, 213, 325-332.

7 P. Tchenio, A. B. Myers and W. E. Moerner, J. Phys. Chem., 1993, 97, 2491-2493.

8 R. M. Dickson, A. B. Cubitt, R. Y. Tsien and W. E. Moerner, Nature, 1997, 388, 355-358.

9 T. Basche and W. E. Moerner, Nature, 1992, 355, 335-337.

10 A. Zumbusch, L. Fleury, R. Brown, J. Bernard and M. Orrit, Phys. Rev. Lett., 1993, 70, 3584-3587.

11 W. P. Ambrose and W. E. Moerner, Nature, 1991, 349, 225-227.

12 F. Schleifenbaum, C. Blum, V. Subramaniam and A. J. Meixner, Mol. Phys., 2009, 107, 1923-1942.

13 H. Wang, A. M. Bardo, M. M. Collinson and D. A. Higgins, J. Phys. Chem. B, 1998, 102, 7231-7237.

14 J. Hofkens, T. Vosch, M. Maus, F. Köhn, M. Cotlet, T. Weil, A. Herrmann, K. Müllen and F. C. D. Schryver, Chem. Phys. Lett., 2001, 333, 255-263.

15 A. Margineanu, J. Hofkens, M. Cotlet, S. Habuchi, A. Stefan, J. Qu, C. Kohl, K. Müllen, J. Vercammen, Y. Engelborghs, T. Gensch and F. C. de Schryver, J. Phys. Chem. B, 2004, 108, 12242-12251.

16 B. Araoz, D. Täuber, C. v. Borczyskowski and P. F. Aramendia, J. Phys. Chem. C, 2012, 116, 7573-7580.

17 M. A. Bopp, A. J. Meixner, G. Tarrach, I. Zschokke-Gränacher and L. Novotny, Chem. Phys. Lett., 1996, 263, 721-726.

18 H. Kobayashi, K. Tsuchiya, K. Ogino and M. Vacha, Phys. Chem. Chem. Phys., 2012, 14, 10114-10118.

19 C. Blum, A. J. Meixner and V. Subramaniam, Biophys. J., 2004, 87, 4172-4179.

20 K. D. Weston, P. J. Carson, H. Metiu and S. K. Buratto, J. Chem. Phys., 1998, 109, 7474-7485.

21 Y. J. Lee, D. Y. Kim and P. F. Barbara, J. Phys. Chem. B, 2006, 110, 9739-9742.

22 S. Chong, W. Min and X. S. Xie, J. Phys. Chem. Lett., 2010, 1, 3316-3322.

23 D. S. English, A. Furube and P. F. Barbara, Chem. Phys. Lett., 2000, 324, 15-19.

24 H. Quante and K. Müllen, Angew. Chem., Int. Ed. Engl., 1995, 34, 1323-1325.

25 M. Celebrano, P. Kukura, A. Renn and V. Sandoghdar, Nat. Photonics, 2011, 5, 95-98.

26 R. Hildner, D. Brinks and N. F. van Hulst, Nat. Phys., 2011, 7, 172-177.

27 J. Hwang, M. M. Fejer and W. E. Moerner, Phys. Rev. A, 2006, 73, 021802.

28 D. Brinks, F. D. Stefani, F. Kulzer, R. Hildner, T. H. Taminiau, Y. Avlasevich, K. Mullen and N. F. van Hulst, Nature, 2010, 465, 905-908.

29 Y. Avlasevich, C. Li and K. Müllen, J. Mater. Chem., 2010, 20, 3814-3826.

30 F. Würthner, Z. Chen, V. Dehm and V. Stepanenko, Chem. Commun., 2006, 1188-1190.

31 L. Piatkowski, E. Gellings and N. F. van Hulst, Broadband single molecule excitation spectroscopy, Nat. Commun., under consideration

32 E. Wientjes, J. Renger, A. G. Curto, R. Cogdell and N. F. v. Hulst, Nat. Commun., 2014, 5, 4236.

33 S. Yampolsky, D. A. Fishman, S. Dey, E. Hulkko, M. Banik, E. O. Potma and V. A. Apkarian, Nat. Photonics, 2014, 8, 650-656. 